\title{
The Biomechanical Properties of a High-Tensile Strength Tape for Tendon Graft Fixation Using the Krackow Configuration
}

\author{
Chih-Kai Hong, M.D., Kai-Lan Hsu, M.D., Fa-Chuan Kuan, M.D., Yueh Chen, M.D., M.Sc., \\ Chen-Hao Chiang, M.D., Ming-Long Yeh, Ph.D., Miin-Jye Wen, Ph.D., and \\ Wei-Ren Su, M.D., M.Sc.
}

\begin{abstract}
Purpose: To compare the biomechanical properties of a high-tensile strength suture with the high-tensile strength tape for tendon graft fixation. Methods: A total of 24 porcine tendons were used and were randomly divided into 2 groups. Two kinds of suture materials, a braided nonabsorbable high-strength suture (group S) and a high-tensile strength tape (group T), were used to complete 3 pairs of Krackow stitches on the tendons. Each specimen was pretensioned to $100 \mathrm{~N}$ for 3 cycles, cyclically loaded from 50 to $200 \mathrm{~N}$ for 200 cycles, and finally loaded to failure. Elongation after cyclic loading, ultimate failure load, and the mode of failure were recorded. Results: The elongation after cyclic loading between group S $(26 \% \pm 5 \%)$ and group T $(24 \% \pm 5 \%)$ were not significantly different $(P=.378)$. The ultimate failure loads in group T $(400 \pm 38 \mathrm{~N})$ were significantly greater than those in group $\mathrm{S}(358 \pm 21 \mathrm{~N})(P=.010)$. All specimens failed because of suture material breakage. Conclusions: Compared with the braided nonabsorbable high-strength suture, the high-tensile strength tape had similar elongation values after cyclic loading, but significantly greater ultimate failure load in this porcine in vitro biomechanical model. Clinical Relevance: A secure suture-tendon construct is especially important when a post-tie fixation technique is used because the mitigating construct may potentially lead to graft loosening and affect graft healing.
\end{abstract}

A secure suture-tendon construct is essential in orthopaedic surgeries, especially in ligament reconstruction surgeries. Once achieving a strong fixation strength, early rehabilitation can be allowed before the completion of biologic healing in the graft tunnel. ${ }^{1-4}$ In past decades, various suture techniques have been addressed for tendon graft fixation ${ }^{1,3-8}$; among these techniques, the Krackow suture ${ }^{6}$ remains the most

From the Department of Orthopaedic Surgery, National Cheng Kung University Hospital, College of Medicine, National Cheng Kung University, Tainan, Taiwan (C-K.H., K-L.H., F-C.K., W-R.S.); Department of Biomedical Engineering, National Cheng Kung University, Tainan, Taiwan (K-L.H., FC.K., C-H.C., M-L.Y.); Department of Orthopaedic Surgery, Sin Lau Hospital, Tainan, Taiwan (Y.C.); Department of Orthopedic Surgery, Chiayi Christian Hospital, Chiayi, Taiwan (C-H.C.); Medical Device Innovation Center, $\mathrm{Na}$ tional Cheng Kung University, Tainan, Taiwan (M-L.Y); Department of Statistics, National Cheng Kung University, Tainan, Taiwan (M-J.W.); and Skeleton Materials and Bio-compatibility Core Lab, Research Center of Clinical Medicine, College of Medicine, National Cheng Kung University (W-R.S.).

Substantial contributions to the conception or design of the work; or the acquisition, analysis, or interpretation of data for the work: C-K.H., K-L.H., F-C.K., M-L.Y., and M-J.W. Drafting the work or revising it critically for important intellectual content: C-K.H., F-C.K., Y.C., and C-H.C. Final approval of the version to be published: K-L.H. and W-R.S. Agreement to be accountable for all aspects of the work in ensuring that questions related to the popular because it provides superior biomechanical properties. $^{9-11}$

In addition to the suture technique, the type of suture material may also affect the fixation strength of a suture-tendon construct. ${ }^{12}$ Gnandt et al. ${ }^{12}$ compared the biomechanical properties of a high-tensile strength tape and a high-tensile strength suture in tendon graft fixation. Their results showed that the high-tensile

accuracy or integrity of any part of the work are appropriately investigated and resolved: $W$-R.S.

The authors report that they have no conflicts of interest in the authorship and publication of this article. Full ICMJE author disclosure forms are available for this article online, as supplementary material.

Received November 17, 2019; accepted May 5, 2020.

Address correspondence to Wei-Ren Su, M.D., M.Sc., Department of Orthopaedic Surgery, National Cheng Kung University Hospital, College of Medicine, National Cheng Kung University, Tainan, Taiwan, No.138, ShengLi Road, Tainan City, Taiwan 70428.E-mail: suwr@ms28.hinet.net

(C) 2020 THE AUTHORS. Published by Elsevier Inc. on behalf of the Arthroscopy Association of North America. This is an open access article under the CC BY-NC-ND license (http://creativecommons.org/licenses/by-nc-nd/4.0/). 2666-061X/191374

https://doi.org/10.1016/j.asmr.2020.05.002 
strength tape withstood a significantly higher load than the high-tensile strength suture during a single load to failure test. ${ }^{12}$

Recently, a high-tensile strength tape, called SutureTape (Arthrex, Naples, FL), was developed to avoid cutting through of soft tissue by the solid cores of sutures as well as creating tears in gloves during surgery. ${ }^{13}$ Unlike the previously proposed high-tensile strength tape, FiberTape (Arthrex). SutureTape does not contain a central ultra-high molecular weight polyethylene round core; instead, the ultra-high molecular weight polyethylene portion extends over the entire width of the tape. ${ }^{13}$ Therefore, 1 of the advantage of the SutureTape is the easiness of making knot-tying, compared with the FiberTape, In addition, the previous biomechanical study indicated that SutureTape featured greater knot security, ultimate load to failure, and tensile stiffness than the high-tensile strength round core suture, FiberWire (Arthrex). ${ }^{13}$

Although the previous biomechanical study indicated that the high-tensile strength tape had greater tendon graft fixation strength than the high-tensile strength suture in a single load to failure test, the impact of a cyclic loading test was not evaluated. ${ }^{12}$ On the other hand, since a high-tensile strength tape was just developed recently, little is known about its biomechanical properties in tendon graft fixation. The purpose of this study was to compare the biomechanical properties of a high-tensile strength suture with the high-tensile strength tape for tendon graft fixation. Our hypothesis was that the suture-tendon construct with the high-tensile strength tape would have a comparable elongation after cyclic loading and a greater maximal failure load.

\section{Methods}

This study was granted an exemption from the institutional review board in our hospital. Porcine flexor profundus tendons were used as surrogates for human semitendinosus tendons. ${ }^{14}$ Tendons of equal length (18 $\mathrm{cm}$ ) were excised from adult male porcine (mean age, 2 years) hindleg trotters. The hindleg trotters were stored at $-20^{\circ} \mathrm{C}$ and thawed to room temperature before dissection.

Next, the entire flexor profundus tendon was harvested and evaluated carefully for possible degenerative or pathologic lesions. After the quality of the tendon was confirmed, the attached soft tissue, such as the synovial sheath, was removed from the tendons. A 5-mm-thick transverse section was acquired from the distal end of each tendon, and the cross-sectional area of each tendon was measured with the assistance of a calibration scale, a digital camera (EOS 60D; Canon, Tokyo, Japan), and open-source image processing software (ImageJ, version 1.52p; National Institutes of Health, Bethesda, MD).
A total of 24 porcine tendons were used and were randomly divided into 2 groups of 12 specimens each. Two kinds of suture materials were selected for the 2 groups, a braided nonabsorbable high-strength suture (group S) and a braided nonabsorbable high-strength tape (group T). In group S, a No. 2 FiberWire (Arthrex) was used, whereas a 1.3-mm SutureTape (Arthrex) was used in group T. Starting $10 \mathrm{~mm}$ from the distal end of the tendon, Krackow stitch configurations with 3 pairs of throws along each side of the tendon were conducted for each specimen. The Krackow stitch began $10 \mathrm{~mm}$ from the distal end of the tendon (Fig 1). To prevent tendon dehydration, $0.9 \%$ saline solution was sprayed on the tendons to keep them moist during preparation and testing.

\section{Biomechanical Testing}

The prepared specimens were mounted on a material-testing system (AG-X; Shimadzu, Tokyo, Japan). The proximal ends of the tendons were fixed with a sinusoid clamp, making the length of the free tendons equal $(9 \mathrm{~cm})$. The ends of the suture limbs were knotted together and looped over a post on the adapter of the material-testing machine (Fig 2). The parameters in the biomechanical testing protocol were in accordance to previous studies. ${ }^{1,4,9,15-21}$ All specimens were initially pretensioned to $100 \mathrm{~N}$ at a rate of $100 \mathrm{~mm} / \mathrm{min}$ for 3 cycles and then preloaded to $50 \mathrm{~N}$ for 1 minute. After that, each specimen was cyclically loaded between 50 and $200 \mathrm{~N}$ with a cross-head speed of $200 \mathrm{~mm} / \mathrm{min}$ for 200 cycles.

A dark blue line was drawn $5 \mathrm{~cm}$ from the distal end of the tendon, whereas another 2 dark blue dots were marked on both suture limbs where they extended from the tendon. These markers served as indicators for measuring the elongation of the suture-tendon construct. By calculating the difference in distance between the dark blue line on the tendon and the marks on both sutures after pretensioning and cyclic loading, the elongation value after cyclic loading could be obtained. A digital camera (HDR-XR 269; Sony, Tokyo, Japan) was used for capturing images, whereas an image processing software (ImageJ, version 1.52p; National Institutes of Health) was used for measuring the distance between the indicators. After completing the cyclic loading, each suture-tendon construct was finally loaded to failure at a rate of $20 \mathrm{~mm} / \mathrm{min}$, where failure was defined as the time at which the maximum tensile force suddenly dropped or was discontinued in the load-displacement curve. The maximal failure load of each specimen was recorded. At the end of the testing, the mode of failure of each specimen was recorded.

\section{Statistical Analysis}

A pilot study with a total of 8 specimens randomly assigned to 2 groups (group $\mathrm{S}$ and group $\mathrm{T}$ ) was 


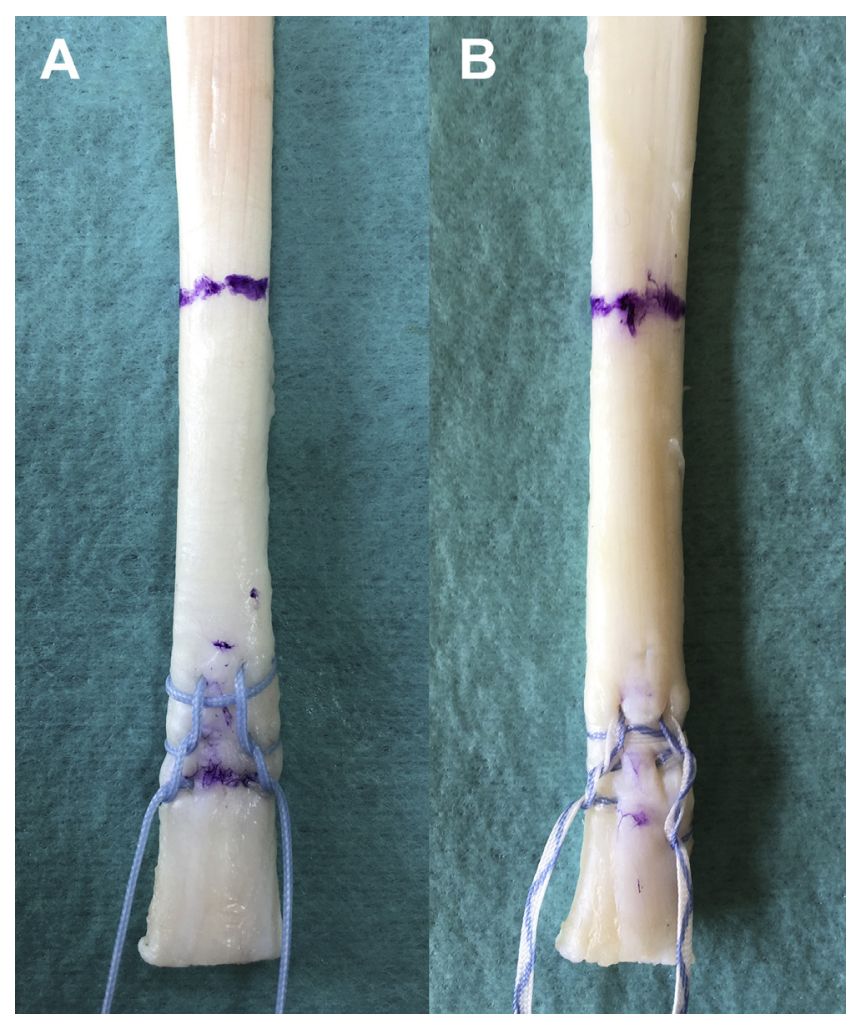

Fig 1. (A) Braided nonabsorbable high-strength suture and (B) high-tensile strength tapes were used to complete 3 pairs of Krackow stitches on the tendons. The Krackow stitch began $10 \mathrm{~mm}$ from the distal end of the tendon.

designed. The data of ultimate failure load were collected and used to calculate the required sample size with $G^{*}$ Power, version 3.1.3, software (Heinrich HeineUniversity of Dusseldorf, Dusseldorf, Germany). After setting an $\alpha$ equal to 0.05 and a power ( $1-\beta$ ) of 0.80 for this a priori power analysis model, an effect size of 0.67 was obtained, and consequently the required sample size for 20 specimens was determined. We finally included 24 samples in total, with 12 in each group.

The statistical analysis was conducted with SPSS for Windows (version 20.0; IBM SPSS Inc., Chicago, IL). Descriptive statistics, such as means and standard deviations, were obtained for both groups. The Wilcoxon signed-rank test, a nonparametric statistical hypothesis test, was used to compare the between-group parameters. The statistical significance was set as $P<.05$.

\section{Results}

The cross-sectional areas of the porcine tendons in group $\mathrm{S}\left(43.4 \pm 3.8 \mathrm{~mm}^{2}\right)$ and group $\mathrm{T}(42.2 \pm 4.6$ $\left.\mathrm{mm}^{2}\right)$ were not significantly different $(P=.443)$. The elongation after cyclic loading in group S $(26 \% \pm 5 \%)$ and group $\mathrm{T}(24 \% \pm 5 \%)$ were not significantly different $(P=.378)$. The ultimate loads to failure in group $\mathrm{T}(400 \pm 38 \mathrm{~N})$ were significantly greater than those in group $\mathrm{S}(358 \pm 21 \mathrm{~N})(P=.010)$. All specimens in both groups failed because of suture or tape breakage at the knot in the final load-to-failure tests (Table 1).

\section{Discussion}

The major findings of this study demonstrated that the high-tensile strength tape group had similar elongation after cyclic loading and significantly greater ultimate failure loads compared with the high-tensile strength suture group. A secure suture-tendon construct is especially important when a post tie fixation technique is used because the mitigating construct may potentially lead to graft loosening and affect graft healing. Numerous biomechanical studies have been conducted to obtain reliable suture-tendon constructs, ${ }^{1,3,4,7,8,11,15-23}$ and using the Krackow suture technique accompanied with a high-tensile strength suture is currently the most popular method. Recently, a high-tensile strength tape was developed, which was reported to have greater knot security, ultimate load to failure, and tensile stiffness as compared

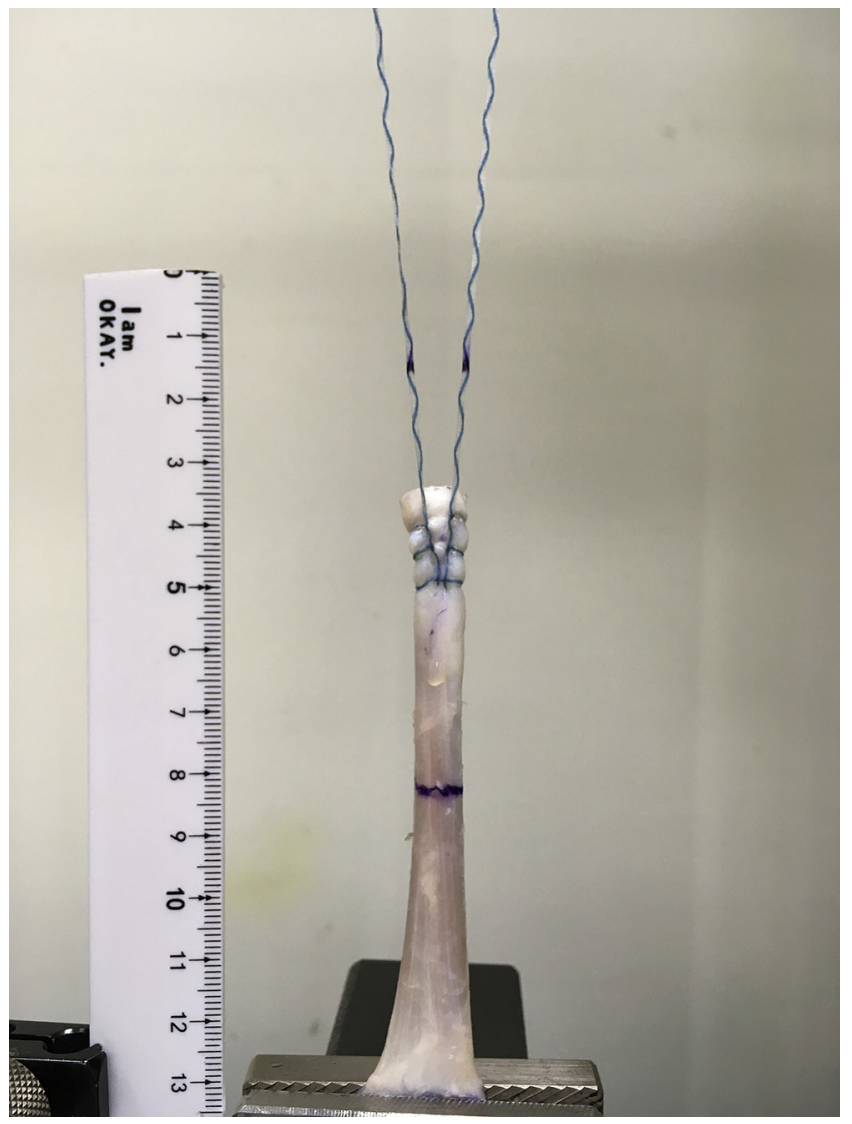

Fig 2. Illustration of the biomechanical testing setup. The tendon was fixed in a sinusoid clamp; the ends of the sutures were knotted tightly and looped over a post on the load cell connected to the materials-testing machine. A dark blue line was drawn $5 \mathrm{~cm}$ from the distal end of the tendon, and 2 dark blue dots was marked on both suture limbs to serve as indicators for measuring the elongation of the suture-tendon construct. 
Table 1. Biomechanical Properties of the Krackow Stitch With High-Strength Sutures (Group S) and High-Strength Tapes (Group T)

\begin{tabular}{|c|c|c|c|}
\hline Groups & $\begin{array}{c}\text { Elongation } \\
\text { After Cyclic } \\
\text { Loading }(\% \pm \mathrm{SD})\end{array}$ & $\begin{array}{l}\text { Ultimate Failure } \\
\text { Load }(\mathrm{N} \pm \mathrm{SD})\end{array}$ & Failure Mode \\
\hline Group S & $26 \% \pm 5 \%$ & $358 \pm 21 \mathrm{~N}^{*}$ & $\begin{array}{l}\text { Rupture of suture } \\
\text { at knot }\end{array}$ \\
\hline Group T & $24 \% \pm 5 \%$ & $400 \pm 38 \mathrm{~N}^{*}$ & $\begin{array}{l}\text { Rupture of suture } \\
\text { at knot }\end{array}$ \\
\hline$P$ value & .378 & .010 & \\
\hline
\end{tabular}

*Significantly different between 2 groups with the Mann-Whitney $U$ test $(P<.05)$.

to the high-tensile strength round core suture. ${ }^{13}$ Our study further indicated the superior biomechanical properties of the high-tensile strength tape for use in tendon graft fixation as compared with a high-tensile strength suture.

The design of tape-shaped suture materials it aimed toward achieving better performance in terms of securing tissue. Theoretically, the larger tissue contact area of a suture tape will allow the force to spread across a broader tissue area, potentially resulting in greater resistance to pull-out forces. ${ }^{12,13}$ A previous biomechanical study seemed to support this viewpoint given that using high-tensile strength tapes in whip stitch and Krackow stitch configurations achieved significantly greater maximum load at failure as compared to using high-tensile strength sutures during a single load to failure test. ${ }^{12}$ When a high-tensile strength tape that is smaller in size, called SutureTape, was developed, Leishman's group evaluated its biomechanical properties and indicated that SutureTape demonstrated superior knot security with greater ultimate load to failure as compared to the high-tensile strength suture. ${ }^{13}$ Our study further evaluated the biomechanical performance of SutureTape in tendon graft fixation. We found that SutureTape had superior biomechanical properties compared with a high-tensile strength suture. Consequently, it is reasonable to consider SutureTape as an alternative in tendon graft fixation.

Given that the cyclic loading test simulates postoperative rehabilitation, larger tendon-graft elongation after cyclic loading may refer to graft loosening, therefore leading to clinical failure. ${ }^{1}$ In the present study, we found $26 \% \pm 5 \%$ and $24 \% \pm 5 \%$ elongation after cyclic loading in the high-tensile strength suture group and high-tensile strength tape group, respectively. The between-group results were not significantly different, indicating that the degree of suture slippage occurring at the suture-tendon junction was similar. Although data from different experiments could not be compared directly, the results from the cyclic loading tests in the present study were consistent with those reported in previous studies. ${ }^{17,20}$ Hong et al. ${ }^{17}$ reported $26.5 \% \pm 3.9 \%$ elongation with the Krackow suture technique in 3 pairs of suture throws using a hightensile strength suture; another study with similar experimental setup also described a $26 \% \pm 6 \%$ elongation after cyclic loading. ${ }^{20}$

Ideally, using tape-shape sutures may provide more contact area at the suture-tendon junction and thus may decrease the pressure on tendons. Thus, using tape-type sutures could potentially decrease the occurrence of tendons pulled through by sutures and enhance resistance to the pull-out forces. A previous biomechanical study agreed with this hypothesis and indicated that standard No. 2 sutures expanded their initial suture holes more than tape-type sutures during cyclic loading tests, thus suggesting that tape-type sutures may be more resistance to suture pulling through tendon. ${ }^{24}$ In the present study, however, suture pulling through tendon was not an issue because all of the specimens failed from suture rupture. Instead, the mechanical strength of the suture itself was found to affect the resultant ultimate failure loads. Because a previous study ${ }^{13}$ demonstrated that high-tensile strength tape has a larger ultimate failure load than a suture, it was not surprising to find a greater ultimate failure load in group $\mathrm{T}$ compared with in group $\mathrm{S}$.

\section{Limitations}

This study had some limitations. First, porcine flexor tendons were used in this study, rather than human tendons. In spite of some structural differences between porcine and human tendons, the porcine flexor tendons are considered to be reasonable surrogates for human semitendinosus tendons. ${ }^{14}$ Second, although a cyclic loading test in a single axial direction was used for simulating repeated movements during rehabilitation, the actual physiologic loading conditions could not be completely simulated. Third, clinical applications could not be completely extrapolated from the results of the present biomechanical study. Despite this, the data from previous reports could be a reference. For example, the mean generated isometric force of quadriceps muscles during an active straight-leg-raising maneuver in a sitting position was around 60 to 70 N. ${ }^{25}$ Finally, the individual surgeon's technique could affect the elongation of the suture-tendon construct. In the current study, all specimens were completed by a single surgeon, thereby reducing operational error.

\section{Conclusions}

Compared with the braided nonabsorbable highstrength suture, the high-tensile strength tape had similar elongation values after cyclic loading, but significantly greater ultimate failure load in this porcine in vitro biomechanical model. 


\section{Acknowledgments}

The authors thank Skeleton Materials and Biocompatibility Core Lab, Research Center of Clinical Medicine, National Cheng Kung University Hospital for the assistance of this project.

\section{References}

1. Deramo DM, White KL, Parks BG, Hinton RY. Krackow locking stitch versus nonlocking premanufactured loop stitch for soft-tissue fixation: A biomechanical study. Arthroscopy 2008;24:599-603.

2. Harvey A, Thomas NP, Amis AA. Fixation of the graft in reconstruction of the anterior cruciate ligament. $J$ Bone Joint Surg Br 2005;87:593-603.

3. McKeon BP, Heming JF, Fulkerson J, Langeland R. The Krackow stitch: A biomechanical evaluation of changing the number of loops versus the number of sutures. Arthroscopy 2006;22:33-37.

4. Su WR, Chu CH, Lin CL, Lin CJ, Jou IM, Chang CW. The modified finger-trap suture technique: A biomechanical comparison of a novel suture technique for graft fixation. Arthroscopy 2012;28:702-710.

5. Hong CK, Chang $\mathrm{CH}$, Chiang CH, Jou IM, Su WR. Hamstring graft preparation using a modified rolling hitch technique. Arthrosc Tech 2014;3:e321-e324.

6. Krackow KA, Thomas SC, Jones LC. A new stitch for ligament-tendon fixation. Brief note. J Bone Joint Surg Am 1986;68:764-766.

7. Krappinger D, Kralinger FS, El Attal R, Hackl W, Haid C. Modified Prusik knot versus whipstitch technique for soft tissue fixation in anterior cruciate ligament reconstruction: A biomechanical analysis. Knee Surg Sports Traumatol Arthrosc 2007; 15:418-423.

8. Wilson L, Banks T, Luckman P, Smith B. Biomechanical evaluation of double Krackow sutures versus the threeloop pulley suture in a canine gastrocnemius tendon avulsion model. Aust Vet J 2014;92:427-432.

9. Barber FA, Howard MS, Piccirillo J, Spenciner DB. A Biomechanical comparison of six suture configurations for soft tissue-based graft traction and fixation. Arthroscopy 2019;35:1163-1169.

10. Michel PA, Domnick C, Raschke MJ, et al. Soft tissue fixation strategies of human quadriceps tendon grafts: A biomechanical study. Arthroscopy 2019;35:3069-3076.

11. Sakaguchi K, Tachibana Y, Oda H. Biomechanical properties of porcine flexor tendon fixation with varying throws and stitch methods. Am J Sports Med 2012;40: $1641-1645$.

12. Gnandt RJ, Smith JL, Nguyen-Ta K, McDonald L, LeClere LE. High-tensile strength tape versus high-tensile strength suture: A biomechanical study. Arthroscopy 2016;32:356-363.
13. Leishman DJ, Chudik SC. Suture tape with broad fullwidth core versus traditional round suture with round core: A mechanical comparison. Arthroscopy 2019;35: 2461-2466.

14. Domnick C, Wieskotter B, Raschke MJ, et al. Evaluation of biomechanical properties: Are porcine flexor tendons and bovine extensor tendons eligible surrogates for human tendons in in vitro studies? Arch Orthop Trauma Surg 2016;136:1465-1471.

15. Hahn JM, Inceoglu S, Wongworawat MD. Biomechanical comparison of Krackow locking stitch versus nonlocking loop stitch with varying number of throws. Am J Sports Med 2014;42:3003-3008.

16. Hong CK, Kuo TH, Yeh ML, Jou IM, Lin CL, Su WR. Do needleless knots have similar strength as the Krackow suture? An in vitro porcine tendon study. Clin Orthop Relat Res 2017;475:552-557.

17. Hong CK, Lin CL, Chang CH, Jou IM, Su WR. Effect of the number of suture throws on the biomechanical characteristics of the suture-tendon construct. Arthroscopy 2014;30:1609-1615.

18. Hong CK, Lin CL, Kuan FC, Wang PH, Yeh ML, Su WR. Longer stitch interval in the Krackow stitch for tendon graft fixation leads to poorer biomechanical property. J Orthop Surg (Hong Kong) 2018;26:2309499018799514.

19. Hong CK, Lin CL, Kuan FC, Wang PH, Yeh ML, Su WR. A biomechanical evaluation of various double Krackow suture techniques for soft-tissue graft fixation. Arthroscopy 2018;34:663-668.

20. Hong CK, Yeh ML, Jou IM, Lin CL, Chang CH, Su WR. Evaluation of 3 needleless grasping suture techniques for soft-tissue graft fixation: A porcine biomechanical study. Arthroscopy 2015;31:1151-1155.

21. White KL, Camire LM, Parks BG, Corey WS, Hinton RY. Krackow locking stitch versus locking premanufactured loop stitch for soft-tissue fixation: A biomechanical study. Arthroscopy 2010;26:1662-1666.

22. Benthien RA, Aronow MS, Doran-Diaz V, Sullivan RJ, Naujoks R, Adams DJ. Cyclic loading of Achilles tendon repairs: A comparison of polyester and polyblend suture. Foot Ankle Int 2006;27:512-518.

23. Ostrander RV 3rd, Saper MG, Juelson TJ. A biomechanical comparison of modified Krackow and locking loop suture patterns for soft-tissue graft fixation. Arthroscopy 2016;32:1384-1388.

24. Ono Y, Joly DA, Thornton GM, Lo IKY. Mechanical and imaging evaluation of the effect of sutures on tendons: Tape sutures are protective to suture pulling through tendon. J Shoulder Elbow Surg 2018;27:1705-1710.

25. Mikaili S, Khademi-Kalantari K, Rezasoltani A, Arzani P, Baghban AA. Quadriceps force production during straight leg raising at different hip positions with and without concomitant ankle dorsiflexion. J Bodyw Mov Ther 2018;22:904-908. 